УДК $517.55+517.923+514.74$

\title{
On Integration of Functions of Complexity One
}

Valery K. Beloshapka*

Faculty of Mechanics and Mathematics

Moscow State University

Vorob'evy Gory, Moscow, 119991

Russia

Received 16.01.2019, received in revised form 04.03.2019, accepted 06.05.2019

We describe functions of complexity one with antiderivative of the same complexity.

Keywords: falsity of analytic functions, complexity classes, integration.

DOI: 10.17516/1997-1397-2019-12-4-496-502.

"Are antiderivatives of some elementary functions still elementary functions?"

Such questions arose at the beginning of differential algebra in the work of Liouville and other authors [1,2]. Here we discuss an analog to this question. In our question, we replace a class of elementary functions with a class of analytical functions of two variables of complexity one: $C l_{1}=\{z(x, y)=c(a(x)+b(y))\}$ (where $(a, b, c)$ are some analytical functions of one variable). This class is the simplest in the hierarchy of complexity classes [3] and the functions of this class have a certain uniqueness property [4].

If a function $z(x, y)$ has a complexity not more than $n<\infty$, then the complexity of its partial derivative $z_{x}(x, y)$ is no more than $2 n$. This is the corollary of the rule of differentiation of a complex function. If in this assumption we replace an inequality with an equality, it will be possible to say that the antiderivative is twice simpler than the original function. But it is possible to get by differentiation of higher complexity function a function of lower complexity. Hence, it is possible that an antiderivative of a function is more complex than a function itself. Though we have some open questions.

Let $N(z)$ be a complexity of the function $z(x, y)$. For an analytical $z$ this number does not depend on the choice of a point and a germ [5].

Question: Is it possible $N(z)<\infty$ and $N\left(\int z(x, y) d x\right)=\infty$ ?

The partial case of this question for $N(z)=1$. Is it possible $N\left(\int c(a(x)+b(y)) d x\right)=\infty$ ?

If $N(z)=0$, the answer is obvious. If $z=a(x)$ then $\int z d x=A(x)+B(y)$ and $N(A+B)=1$. If $z=b(y)$, then $\int z d x=b(y) x+\beta(y)=B(x, y)$ and $N(B) \leqslant 2$.

In this paper, we answer a simpler question. Which functions of complexity one have antiderivatives of the same complexity?

Consider a function of complexity one $z(x, y)=c(a(x)+b(y))$, where $(a, b, c)$ are not constant. By differentiation we get

$$
w(x, y)=z_{x}^{\prime}=c^{\prime}(a(x)+b(y)) a^{\prime}(x) .
$$

The complexity of $w$ is no more than two. Let its complexity be no more than one. It means (see [3])

$$
\delta(w)=\left(\ln \left(\frac{w_{x}^{\prime}}{w_{y}^{\prime}}\right)\right)_{x y}^{\prime \prime}=0
$$

*vkb@strogino.ru

(c) Siberian Federal University. All rights reserved 
and we have

$$
\begin{aligned}
&-c_{4} a_{1}{ }^{2} a_{2} c_{2}{ }^{2} c_{1}+2 a_{1}{ }^{2} c_{3}{ }^{2} a_{2} c_{2} c_{1}-a_{1}{ }^{2} c_{3} a_{2} c_{2}{ }^{3}-a_{1} c_{3} c_{2}{ }^{2} a_{3} c_{1}+a_{1} c_{2}{ }^{4} a_{3}- \\
&-c_{4} a_{2}{ }^{2} c_{2} c_{1}{ }^{2}+c_{3}{ }^{2} a_{2}{ }^{2} c_{1}{ }^{2}+3 c_{3} a_{2}{ }^{2} c_{2}{ }^{2} c_{1}-3 a_{2}{ }^{2} c_{2}{ }^{4}=0
\end{aligned}
$$

This differential polynomial depends on derivatives which we denote subscripts $\left(c^{\prime}, c^{\prime \prime}, c^{\prime \prime \prime}, c^{I V}, a^{\prime}, a^{\prime \prime}, a^{\prime \prime \prime}\right)$. If we replace $b^{-1}(t-a(x))$ with $y$ in $c(a(x)+b(y))$ we will get $c(t)$. That's why we can consider $c, a$ and its derivatives as functions of independent variables $t$ and $x$.

Case $A$. Let $c_{2}=0$, i.e. $c(t)$ is linear. After a change of $a$ and $b$ we can consider $z=a(x)+b(y)$. But in this case the derivative $z_{x}^{\prime}=a^{\prime}(x)$ has a complexity zero.

Case $C$. Let $a_{2}=0$, i.e. $a(x)=k x+l$. After a change of $a$ and $b$ we can consider $a(x)=x$, i.e. $z=c(x+b(y))$. And $z_{x}^{\prime}$ has a complexity one for all non-constant $a$ and $c$.

If $a^{\prime}=a_{1}$ and $c^{\prime}=c_{1}$ are non-constant, then we can consider $A=a_{1}$ and $C=c_{1}$ as independent variables and $P(A)=a_{2}, Q(C)=c_{2}$ as unknown functions. And we can rewrite (2) as

$$
\begin{aligned}
& A^{2} C Q(C) \frac{\mathrm{d}^{2}}{\mathrm{~d} C^{2}} Q(C)-A^{2} C\left(\frac{\mathrm{d}}{\mathrm{d} C} Q(C)\right)^{2}+A^{2} Q(C) \frac{\mathrm{d}}{\mathrm{d} C} Q(C)+ \\
& +A C\left(\frac{\mathrm{d}}{\mathrm{d} C} Q(C)\right) \frac{\mathrm{d}}{\mathrm{d} A} P(A)+C^{2} P(A) \frac{\mathrm{d}^{2}}{\mathrm{~d} C^{2}} Q(C)-A Q(C) \frac{\mathrm{d}}{\mathrm{d} A} P(A)- \\
& \quad-3 C P(A) \frac{\mathrm{d}}{\mathrm{d} C} Q(C)+3 P(A) Q(C)=0
\end{aligned}
$$

For each fixed $C$ the equation (3) is the ordinary differential equation in relation to $P(A)$. This equation has a form

$$
k A \frac{\mathrm{d}}{\mathrm{d} A} P(A)+l P(A)+m A^{2}=0
$$

where

$$
\begin{aligned}
& k=C \frac{\mathrm{d}}{\mathrm{d} C} Q(C)-Q(C), \\
& l=C^{2} \frac{\mathrm{d}^{2}}{\mathrm{~d} C^{2}} Q(C)-3 C \frac{\mathrm{d}}{\mathrm{d} C} Q(C)+3 Q(C), \\
& m=C Q(C) \frac{\mathrm{d}^{2}}{\mathrm{~d} C^{2}} Q(C)-C\left(\frac{\mathrm{d}}{\mathrm{d} C} Q(C)\right)^{2}+\left(\frac{\mathrm{d}}{\mathrm{d} C} Q(C)\right) Q(C) .
\end{aligned}
$$

Case $B$. Let $k=0$. As a solution to this equation we get $Q(C)=\lambda C$ and then $c(t)=$ $=(\mu / \lambda) \exp (\lambda t)+\nu$. And we see that in this case $z$ has a form $z=a(x) b(y)$. It is clear that $z_{x}^{\prime}$ has a complexity one for all non-constant $a$ and $b$.

Let $G$ be a pseudo-group of transformations of the form

$$
\begin{aligned}
& a(x) \rightarrow a(\alpha x+\tilde{\alpha}), \\
& b(y) \rightarrow b(\beta(y)), \\
& c(t) \rightarrow \gamma c(t)+\tilde{\gamma} .
\end{aligned}
$$

for all constants $\alpha \neq 0, \tilde{\alpha}, \gamma \neq 0, \tilde{\gamma}$ and for any non-constant $\beta(y)$. And let $H$ be a (pseudo)subgroup of $G$ of transformations of the form

$$
\begin{aligned}
& a(x) \rightarrow a(x), \\
& b(y) \rightarrow b(\beta(y)), \\
& c(t) \rightarrow \gamma c(t)+\tilde{\gamma} .
\end{aligned}
$$


Statement: (a) An analytical function $z(x, y)=c(a(x)+b(y))((a, b, c)$ are non-constant) has a derivative $z_{x}^{\prime}(x, y)=c^{\prime}(a(x)+b(y)) a^{\prime}(x)$ of complexity one if and only if it is true for $Z=g \circ z$, where $g \in G$.

(b) A function $z(x, y)=c(x b(y))\left((b, c)\right.$ are non-constant) has a derivative $z_{x}^{\prime}(x, y)=$ $=c^{\prime}(x b(y)) b(y)$ of a complexity one if and only if it is true for $Z=h \circ z$, где $h \in H$.

The proof we will get by the differentiation.

Case 1 . Let $l=0, k \neq 0$. From the equation $l=0$ we have

$$
Q(C)=C^{3} \lambda+C \mu .
$$

From $k \neq 0$ we get $\lambda \neq 0$

Case 1.1 (in the theorem, this case is denoted as(1)). Let $\mu=0$ then we get

$$
c(t)=-\frac{\sqrt{-2 \lambda t+\nu}}{\lambda}, \quad a(x)=\tau x^{2}+\kappa x+\rho .
$$

It is possible to transform function $z=c(a(x)+b(y))$ in

$$
z=\sqrt{x^{2}+y^{2}} \text {, and its derivative in } z_{x}^{\prime}=\frac{x}{\sqrt{x^{2}+y^{2}}}
$$

by transformations from $G$.

Case 1.2. Let $\mu \neq 0$ then we get

$$
c^{\prime}(t)=\frac{\sqrt{\left(\nu \mu \mathrm{e}^{-2 \mu t}-\lambda\right) \mu}}{\nu \mu \mathrm{e}^{-2 \mu t}-\lambda}
$$

and

$$
c(t)=-\frac{1}{\sqrt{\lambda \mu}} \arctan \left(\frac{\sqrt{\nu \mu^{2} \mathrm{e}^{-2 \mu t}-\lambda \mu}}{\sqrt{\lambda \mu}}\right)
$$

By substitution (5) into (3) we get

$$
2 A \mu+\frac{\mathrm{d}}{\mathrm{d} A} P(A)=0
$$

then $P(A)=-A^{2} \mu-\nu^{2}$ or

$$
\frac{\mathrm{d}^{2}}{\mathrm{~d} x^{2}} a(x)+\mu\left(\frac{\mathrm{d}}{\mathrm{d} x} a(x)\right)^{2}+\nu^{2}=0 .
$$

If $\nu=0$ then by integration we get

$$
a(x)=\frac{\ln (\mu \kappa x+\mu \rho)}{\mu}
$$

(in the theorem, this case is denoted as (2)). By transformations from $G$ we can transform this $z=c(a(x)+b(y))$ into

$$
z=\arctan \left(\frac{\sqrt{-x^{2} y^{2}+1}}{x y}\right), \text { and its derivative into } z_{x}^{\prime}=-\frac{y}{\sqrt{-x^{2} y^{2}+1}} .
$$

If $\nu \neq 0$ then by integration we get

$$
a(x)=\frac{1}{2 \mu} \ln \left(\frac{\mu(\kappa \sin (\nu \sqrt{\mu} x)-\rho \cos (\nu \sqrt{\mu} x))^{2}}{\nu^{2}}\right)
$$


(in the theorem, this case is denoted as (3)). By transformations from $G$ we can transform such function $z=c(a(x)+b(y))$ into

$$
z=\arctan \left(\frac{\sqrt{1-(\sin (x))^{2} y^{2}}}{\sin (x) y}\right) \text {, and its derivative in } z_{x}^{\prime}=-\frac{y \cos (x)}{\sqrt{1-(\sin (x))^{2} y^{2}}} .
$$

Case 2 (in the theorem, this case is denoted as (I)). Let $k \neq 0, l \neq 0, l+2 k=0$ then from equation $l+2 k=0$ we get

$$
Q(C)=\lambda C+\mu C \ln (C) .
$$

If $\mu=0$ then $Q(C)=\lambda C$ and $k=0$. From this contradiction we get $\mu \neq 0$. Now we have $Q(C)=\lambda C+\mu C \ln (C)$, i.e. $c(t)$ satisfies the equation

$$
c^{\prime \prime}(t)=c^{\prime}(t)\left(\lambda+\mu \ln \left(c^{\prime}(t)\right)\right) .
$$

After substitution this expression for $Q(C)$ into (3) we get

$$
A^{2} \mu-A \frac{\mathrm{d}}{\mathrm{d} A} P(A)+2 P(A)=0
$$

and then we get $P(A)=(\mu \ln (A)+\nu) A^{2}$. Then we solve the equation

$$
a^{\prime \prime}(x)=\left(\mu \ln \left(a^{\prime}(x)\right)+\nu\right) a^{\prime}(x)^{2}
$$

and get $a(x)$.

Case 3. Let $k \neq 0, l \neq 0, l+2 k \neq 0$. From equation (4) we get

$$
P(A)=-\frac{m A^{2}}{2 k+l}+A^{-\frac{l}{k}} n
$$

or

$$
\frac{\mathrm{d}^{2}}{\mathrm{~d} x^{2}} a(x)+\frac{m\left(\frac{\mathrm{d}}{\mathrm{d} x} a(x)\right)^{2}}{2 k+l}-\left(\frac{\mathrm{d}}{\mathrm{d} x} a(x)\right)^{-\frac{l}{k}} n=0 .
$$

Case 3.1 (in the theorem, this case is denoted as (4)). Let $n=0$ then $P(A)=-\frac{m A^{2}}{2 k+l}$ and we get

$$
a(x)=-\frac{2 k+l}{m} \ln (x)+\rho .
$$

Now we can represent $z$ as $z=c(x b(y))$. Let us write the first class condition (1) for $w=z_{x}^{\prime}=$ $=c^{\prime}(x b(y)) b(y)$. Then after substitution $x=t / b(y)$ we get

$$
\begin{aligned}
c_{3} c_{1}{ }^{2} c_{0} t^{2}-2 c_{1} c_{0} c_{2}{ }^{2} t^{2}+c_{1}{ }^{3} c_{2} t^{2} & +c_{3} c_{1} c_{0}{ }^{2} t-c_{0}{ }^{2} c_{2}{ }^{2} t- \\
& -2 c_{1}{ }^{2} c_{0} c_{2} t+2 c_{1}{ }^{4} t+c_{1} c_{0}{ }^{2} c_{2}-2 c_{1}{ }^{3} c_{0}=0 .
\end{aligned}
$$

Then from this equation we get

$$
c(t)=\frac{\mathrm{e}^{\mu} \nu}{t}\left(t^{-\lambda^{-1}} \mathrm{e}^{\frac{\mu}{\lambda}}-1\right)^{-\lambda}
$$

By transformation from $H$ we can transform such function $z=c(x b(y))$ into

$$
z=\left(1+(x y)^{m}\right)^{(1 / m)} \text {, а ее производную в } z_{x}^{\prime}=\frac{\left(1+(x y)^{m}\right)^{(1 / m)}(x y)^{m}}{x\left(1+(x y)^{m}\right)} .
$$


Case 3.2 (in the theorem, this case is denoted as (II)). Let $n \neq 0$ then we can substitute (6) into (2), separate the terms with a multiplier $A^{2}$ and the terms with a multiplier $A^{-\frac{l}{k}}$. We have two equations

$$
\begin{gathered}
-C^{2} k m q_{2}+2 C k^{2} q_{0} q_{2}-2 C k^{2} q_{1}^{2}+C k l q_{0} q_{2}-C k l q_{1}^{2}+C k m q_{1}+2 k^{2} q_{0} q_{1}+k l q_{0} q_{1}-k m q_{0}=0, \\
2 C^{2} k^{2} n q_{2}+C^{2} k l n q_{2}-6 C k^{2} n q_{1}-5 C k l n q_{1}-C l^{2} n q_{1}+6 k^{2} n q_{0}+5 k l n q_{0}+l^{2} n q_{0}=0 .
\end{gathered}
$$

By elimination of $q_{2}$ from this equations we get (see our inequalities)

$$
C\left(\frac{\mathrm{d}}{\mathrm{d} C} Q(C)\right) k+C m-3 Q(C) k-Q(C) l=0 .
$$

From this equation we get

$$
Q(C)=\frac{C m}{2 k+l}+C^{3+\frac{l}{k}} \lambda .
$$

This equation with (6) transforms (3) into identity. This expression for $Q(A)$ is equivalent to the equation

$$
\frac{\mathrm{d}^{2}}{\mathrm{~d} t^{2}} c(t)-\frac{m \frac{\mathrm{d}}{\mathrm{d} t} c(t)}{2 k+l}-\left(\frac{\mathrm{d}}{\mathrm{d} t} c(t)\right)^{3+\frac{l}{k}} \lambda=0 .
$$

So, we have proven our main theorem.

Theorem. Let an analytical function of a complexity one $z(x, y)$ have a derivative of a complexity no more than one $z_{x}^{\prime}(x, y)$. Then the function is one of the following list:

$$
\begin{aligned}
& (A) \quad z=a(x)+b(y), \quad z_{x}^{\prime}=a^{\prime}(x), \\
& (B) \quad z=a(x) b(y), \quad z_{x}^{\prime}=a^{\prime}(x) b(y), \\
& (C) \quad z=c(x+b(y)), \quad z_{x}^{\prime}=c^{\prime}(x+b(y))
\end{aligned}
$$

or the function $z=c(a(x)+b(y))$ up to transformations of $G$ has the following form

$$
\begin{aligned}
& \text { (1) } z=\sqrt{x^{2}+y^{2}}, \quad z_{x}^{\prime}=\frac{x}{\sqrt{x^{2}+y^{2}}}, \\
& \text { (2) } z=\arctan \left(\frac{\sqrt{-x^{2} y^{2}+1}}{x y}\right), \quad z_{x}^{\prime}=-\frac{y}{\sqrt{-x^{2} y^{2}+1}}, \\
& \text { (3) } z=\arctan \left(\frac{\sqrt{1-(\sin (x))^{2} y^{2}}}{\sin (x) y}\right), \quad z_{x}^{\prime}=-\frac{y \cos (x)}{\sqrt{1-(\sin (x))^{2} y^{2}}}, \\
& \text { (4) } z=\left(1+(x y)^{m}\right)^{(1 / m)}, \quad z_{x}^{\prime}=\frac{\left(1+(x y)^{m}\right)^{(1 / m)}(x y)^{m}}{x\left(1+(x y)^{m}\right)}
\end{aligned}
$$

or the function has a form $z=c(a(x)+b(y))$, where $b(y)$ is arbitrary and $(c(t), a(x))$ are any of the solutions of the following equations

$$
\begin{aligned}
& \text { (I) } \quad c^{\prime \prime}(t)=\left(\mu \ln \left(c^{\prime}(t)\right)+m\right) c^{\prime}(t), \quad a^{\prime \prime}(x)=\left(\mu \ln \left(a^{\prime}(x)\right)+n\right) a^{\prime}(x)^{2}, \\
& (I I) \quad c^{\prime \prime}(t)-\lambda c^{\prime}(t)+\nu\left(c^{\prime}(t)\right)^{3+\mu}=0, \quad a^{\prime \prime}(x)+\lambda\left(a^{\prime}(x)\right)^{2}+n\left(a^{\prime}(x)\right)^{-\mu}=0 .
\end{aligned}
$$

In both of these cases $z_{x}^{\prime}=c^{\prime}(a(x)+b(y)) a^{\prime}(x)$ has a complexity one. 
Remark 1. In cases (I) and (II) it is possible to represent the functions $c(t)$ and $a(x)$ in quadratures. In the case (I) $c$ and $a$ have a representation in a form of an exponential integral $\operatorname{Ei}(p, s)$. In the case(II) we have a representation in a form of a hypergeometric function ${ }_{2} \mathrm{~F}_{1}(\alpha, \beta ; \gamma ; s)$.

Let us consider the equations from the case (I). With the use of the transformation $c(t) \rightarrow$ $\gamma c(t)$ we can make $m=0$ in the first equation. With the use of the transformation $a(x) \rightarrow a(\alpha x)$ we can make $n=0$ in the second equation. From the equation

$$
c^{\prime \prime}(t)=\mu c^{\prime}(t) \ln \left(c^{\prime}(t)\right)
$$

we get

$$
c(t)=-\frac{E i\left(1, \rho \mathrm{e}^{\mu t}\right)}{\mu}+\sigma
$$

where

$$
E i(p, s)=\int_{1}^{\infty} e^{-t s} t^{-p} d t
$$

is the exponential integral. Then we can represent $a(x)$ with the same $\operatorname{Ei}(p, s)$ (and by the inverse function and integration).

Let us consider the equations from the case (II). From the first equation we get

$$
c(t)=\int\left(\frac{\lambda}{\nu \mathrm{e}^{\rho \lambda \mu} \mathrm{e}^{\lambda \mu t}\left(\mathrm{e}^{\rho \lambda}\right)^{2}\left(\mathrm{e}^{\lambda t}\right)^{2}+1}\right)^{(\mu+2)^{-1}} \mathrm{e}^{\rho \lambda} \mathrm{e}^{\lambda t} \mathrm{~d} t+\sigma .
$$

It is clear that this function is the composition of the exponent and the integral of the form

$$
\int \frac{d \sigma}{\left(\Lambda \sigma^{2}+1\right)^{\frac{1}{\mu+2}}}=\sigma{ }_{2} \mathrm{~F}_{1}\left(1 / 2,(\mu+2)^{-1} ; 3 / 2 ;-\Lambda \sigma^{2}\right),
$$

This integral is a hypergeometric function. We may express the function $a(x)$ from the second equation with the use of the inverse function and integration

$$
\int \frac{d \sigma}{\lambda \sigma^{2}+n \sigma^{-\mu}}
$$

After transformation $\sigma \rightarrow \frac{\rho}{\sigma}$ this integral transforms into the integral of the form

$$
\int \frac{d \sigma}{\sigma^{2-\mu}+1}=\sigma_{2} \mathrm{~F}_{1}\left(1,(2-\mu)^{-1} ; 1+(2-\mu)^{-1} ;-\sigma^{2-\mu}\right) .
$$

This integral is a hypergeometric function.

Remark 2. The cases (A), (B), (C) and (1), (2), (3), (4) have very explicit descriptions. Descriptions of the cases (I) and (II) are a little bit more complicated.

Remark 3. The description in our theorem has the following format: (function, its derivative). By transposition of this pair we get a description of the following format: (function, antiderivative).

The research was financially supported by RFBR grants 17-01-00592 a and 18-51-41011 Uzb $t$.

\section{References}

[1] J.Liouville, Sur la détermination des intégrales dont la valeur est algébrique, Journal de l'ecole polytechnique, XIV(1833), Sec. 23. 
[2] J.F.Ritt, Integration in finite term, Liouville's theory of elementary methods, NY, Colombia University Press, 1948.

[3] V.K.Beloshapka, Analytic Complexity of Functions of Two Variables, Russ. J. Math. Phys., 14(2007), no. 3, 243-249.

[4] V.K.Beloshapka, Algebraic Functions of Complexity One, a Weierstrass Theorem, and Three Arithmetic Operations, Russian Journal of Mathematical Physics, 23(2016), no. 3, 343-347.

[5] V.K.Beloshapka, Decomposition of Functions of Finite Analytical Complexity, Journal of Siberian Federal University. Mathematics Physics, 11(2018), no. 6, 680-685.

\section{Об интегрировании функций сложности 1}

Валерий К. Белошапка

Механико-математический факультет МГУ им. Ломоносова

Воробьевы горы, Москва, 119991

Россия

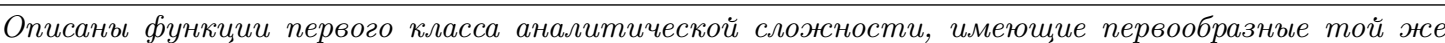
сложности.

Ключевые слова: сложность аналитических функиий, классы сложности, интегрирование. 